https://helda.helsinki.fi

\title{
Bowel Biofilms : Tipping Points between a Healthy and Compromised Gut?
}

\section{Tytgat, Hanne L. P.}

2019-01

Tytgat , H L P , Nobrega , F L , van der Oost , J \& de Vos , W M 2019 , ' Bowel Biofilms :

Tipping Points between a Healthy and Compromised Gut? ' , Trends in Microbiology , vol. 27 , no. 1 , pp. 17-25 . https://doi.org/10.1016/j.tim.2018.08.009

http://hdl.handle.net/10138/310697

https://doi.org/10.1016/j.tim.2018.08.009

publishedVersion

Downloaded from Helda, University of Helsinki institutional repository.

This is an electronic reprint of the original article.

This reprint may differ from the original in pagination and typographic detail.

Please cite the original version. 


\title{
Bowel Biofilms: Tipping Points between a Healthy and Compromised Gut?
}

\author{
Hanne L.P. Tytgat (D), ${ }^{1,2, \star}$ Franklin L. Nobrega, ${ }^{1,3}$ John van der Oost, ${ }^{1}$ and Willem M. de Vos ${ }^{1,4, *}$
}

Bacterial communities are known to impact human health and disease. Mixed species biofilms, mostly pathogenic in nature, have been observed in dental and gastric infections as well as in intestinal diseases, chronic gut wounds and colon cancer. Apart from the appendix, the presence of thick polymicrobial biofilms in the healthy gut mucosa is still debated. Polymicrobial biofilms containing potential pathogens appear to be an early-warning signal of developing disease and can be regarded as a tipping point between a healthy and a diseased state of the gut mucosa. Key biofilm-forming pathogens and associated molecules hold promise as biomarkers. Criteria to distinguish microcolonies from biofilms are crucial to provide clarity when reporting biofilm-related phenomena in health and disease in the gut.

\section{Bacteria Like to Form Biofilms}

Bacterial biofilms (see Glossary) are as ubiquitous as bacteria. Defined as matrix-enclosed mixed populations of bacteria and/or archaea (the focus here is on bacteria) that adhere to biotic and abiotic surfaces, biofilms are communities in which the microorganisms closely collaborate as a strategy for survival and persistence [1] (Box 1). Biofilms initially develop when bacteria attach to a surface and form small aggregates of bacteria. A mature biofilm forms when these microcolonies embed themselves in a complex self-produced matrix of secreted polysaccharides. At some stage, bacterial cells can disperse from this mature biofilm to colonize new niches [1,2] (Box 1).

Biofilms offer their microbial inhabitants many competitive advantages that vary from efficient nutrient exchange to increased stress resistance [3]: they form the ideal environment for crossfeeding and the establishment of a digestive consortium [2], and they help bacteria to withstand biological, chemical, and physical stresses [4]. The strength of the interactions in biofilms fits the Black Queen hypothesis: bacteria may lose the ability to perform certain essential functions by relying heavily on other species in close proximity [5]. Biofilms furthermore promote horizontal gene transfer through the exchange of bacterial genome fragments and/or mobile genetic elements, which, for instance, contributes to spreading of antibiotic-resistance genes [6]. The extreme tolerance of biofilms to antibiotic and antimicrobial substances is particularly cumbersome as this complicates fighting pathogens, the more so when they are resistant to antibiotics [6]. In the context of microbial-host interactions, biofilms offer bacteria a protective niche that helps them to evade host defense. Biofilms can thus play an important role in pathogenesis. The intimate contact of bacterial consortia with the host is also linked to the capacity of biofilms to promote synergy between both partners, stimulating nutrient digestion, and even fortifying host defense systems [7]. In addition, bacterial biofilms that develop on food particles in the colon lumen are known to contribute to nutrient processing [8].

One of the niches in which microbial biofilms are widely studied is the orogastrointestinal tract of the human body. Here biofilms have most commonly been associated with disease, including dental plaque [9], stomach infections [10], inflammatory bowel disease (IBD) [11],

\section{Highlights}

Bacteria occur in a polymicrobial biofilm in stressful niches, which offers many competitive advantages (e.g., nutrient and gene exchange) and protection from stressors.

In contrast to oral biofilms, the occurrence and features of healthy gastrointestinal mucosal biofilms, if any, are poorly understood. This pertains to the difficulty in sampling the gastrointestinal tract of healthy persons and the distinctive biogeography and physiology of animal models.

Adhesion, microcolony, and biofilm formation are different points on a continuum describing increasing complexity of colonizing bacterial communities. However, all that adheres is not biofilm.

The establishment of mature polymicrobial pathogenic biofilms might be an early-warning signal of the shift from a healthy towards a diseased microbiota. Driver species and key metabolites offer potential novel biomarkers.

${ }^{1}$ Laboratory of Microbiology, Wageningen University, 6708 WE Wageningen, The Netherlands ${ }^{2}$ Institute of Microbiology, Swiss Federal Institute of Technology, ETH Zurich, 8093 Zurich, Switzerland ${ }^{3}$ Kavli Institute of Nanoscience and Department of BioNanoscience, Delft University of Technology, $2629 \mathrm{HZ}$ Delft, The Netherlands ${ }^{4}$ Faculty of Medicine, Immunobiology Research Program, Department of Bacteriology and Immunology, University of Helsinki, 00290 Helsinki, Finland

*Correspondence: hanne.tytgat@wur.nl (H.L.P. Tytgat) and willem.devos@wur.nl (W.M.de Vos). 


\section{Box 1. All That Adheres Is Not Biofilm}

Crucial, but often neglected, is the distinction between biofilms, microcolonies, and other adhesion events. These three phenomena form parts of a continuum of increasing community complexity (Figure I). The three phenomena are closely intertwined, but not interchangeable nor synonymous.

Adhesion describes the event in which the bacteria initiate contact with their environment via their cell envelope molecules and appendages, such as pili and flagella. After initial contact, a multitude of interactions between ligands and receptors on the surface of both the bacteria and the host surface strengthen the interaction. Adhesion is crucial in bacterial colonization, and a crucial first step in the formation of microcolonies and biofilm.

Microcolonies are small aggregates of adhering bacteria that grow together when environmental conditions are suboptimal, resulting in a fitness advantage over planktonic growth. Often they are covered in a simple, protective matrix [55]. These bacterial consortia form one of the simplest 3D multicellular assemblies in nature. Microcolonies can establish themselves strongly in small environmental niches.

Biofilms are bigger populations of bacteria embedded in a thick, complex, self-produced matrix often containing multiple species [1,2]. The close contact between the members of a biofilm drives collective behavior, like cooperation and nutrient exchange. Members of a biofilm communicate using quorum sensing, that is, via the production of chemical messengers. Bacteria in a biofilm have a distinct physiology from planktonic cells, which is reflected in the differential regulation of the expression of several genes. Within biofilms one can discern several bacterial populations: viable and metabolically active, dormant or stationary bacteria, and persister cells.

Although these three terms describe distinct microbial states with associated biological processes, they are all part of the same continuum of increasing community complexity. These properties make the three phenomena hard to distinguish, and a clear, widely accepted cut-off to discriminate between them is lacking. Often, mere adhesion events are reported as biofilm formation, whilst the proper experimental results and controls (e.g., repeated washes to remove loosely associated planktonic bacteria, tests exploring the recalcitrance and resistance of bacteria, differential gene expression analysis) are lacking. There is a need to establish novel methods that allow for the distinction between adhesion events, mostly harmless microcolonies and thick, pathogenic, polymicrobial biofilms breeching the intestinal cell wall. Distinct features like the detection of quorum sensing molecules or altered gene expression can form the basis for novel techniques beyond fluorescence in situ hybridization (FISH) to evaluate colony size [40].

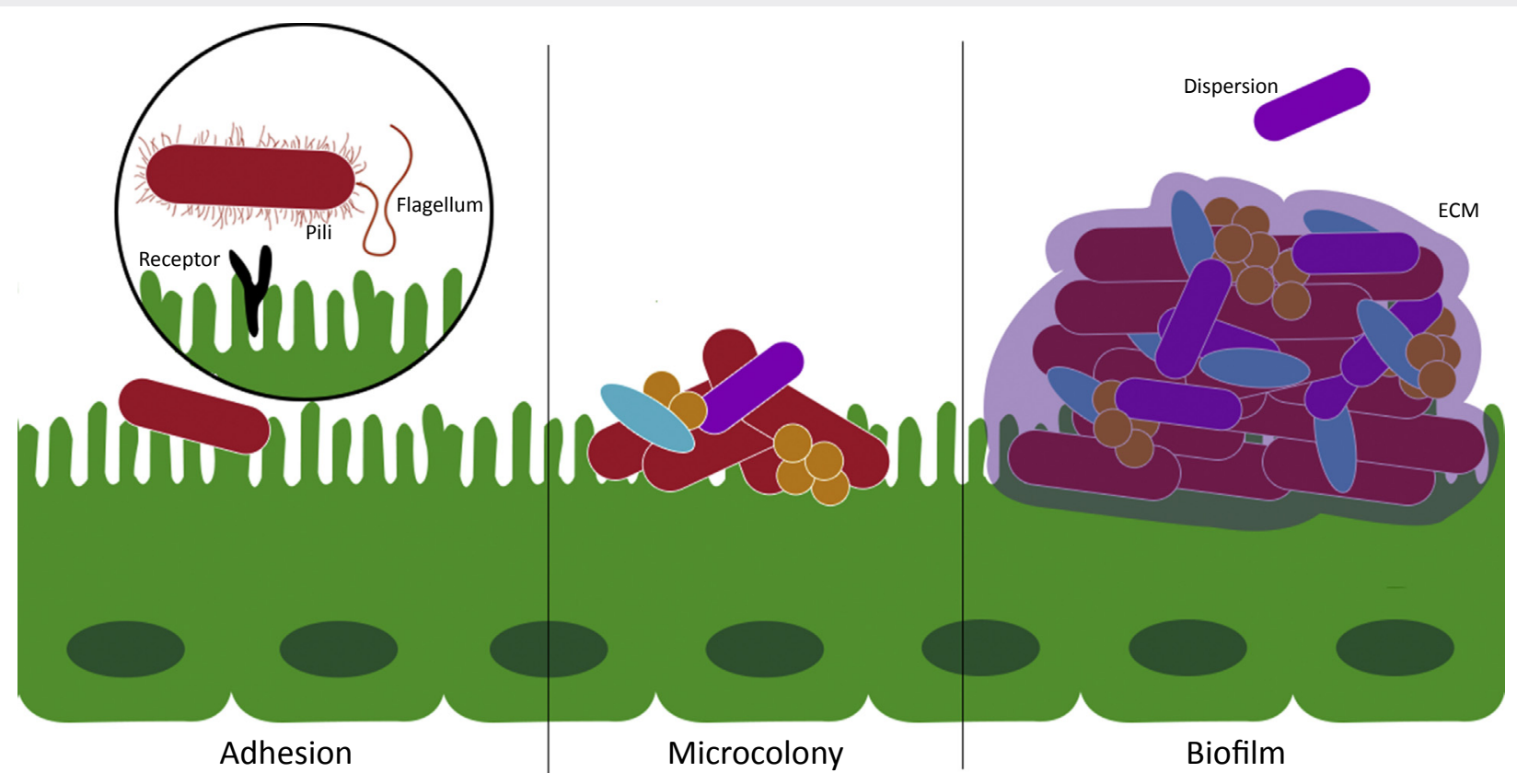

Trends in Microbiology

Figure I. Adhesion versus Microcolonies versus Biofilm.Bacteria adhere to (a)biotic surfaces using their surface appendages (e.g., pili, flagella) to establish initial contact. A microcolony is formed when several bacteria colocalize and protect themselves with a simple matrix. When a community of bacteria grows even bigger and forms a robust multispecies aggregate of bacteria and/or archaea embedded in a thick extracellular matrix, a biofilm is formed. Once a biofilm is established, single cells start to disperse and can colonize new niches. Abbreviations: ECM, extracellular matrix. 
and other infectious diseases [12,13] (Figure 1, Key Figure). So far, healthy biofilms have only been substantiated in the oral cavity (mainly on nonmucosal, solid surfaces) $[14,15]$ and appendix [16] (Box 2). In addition, it has been suggested that the colon microbiota manifests itself as a biofilm $[3,17,18]$. Other studies in contrast describe a role for polymicrobial

\section{Key Figure}

\section{Biofilm Formation throughout the Orogastrointestinal Tract}

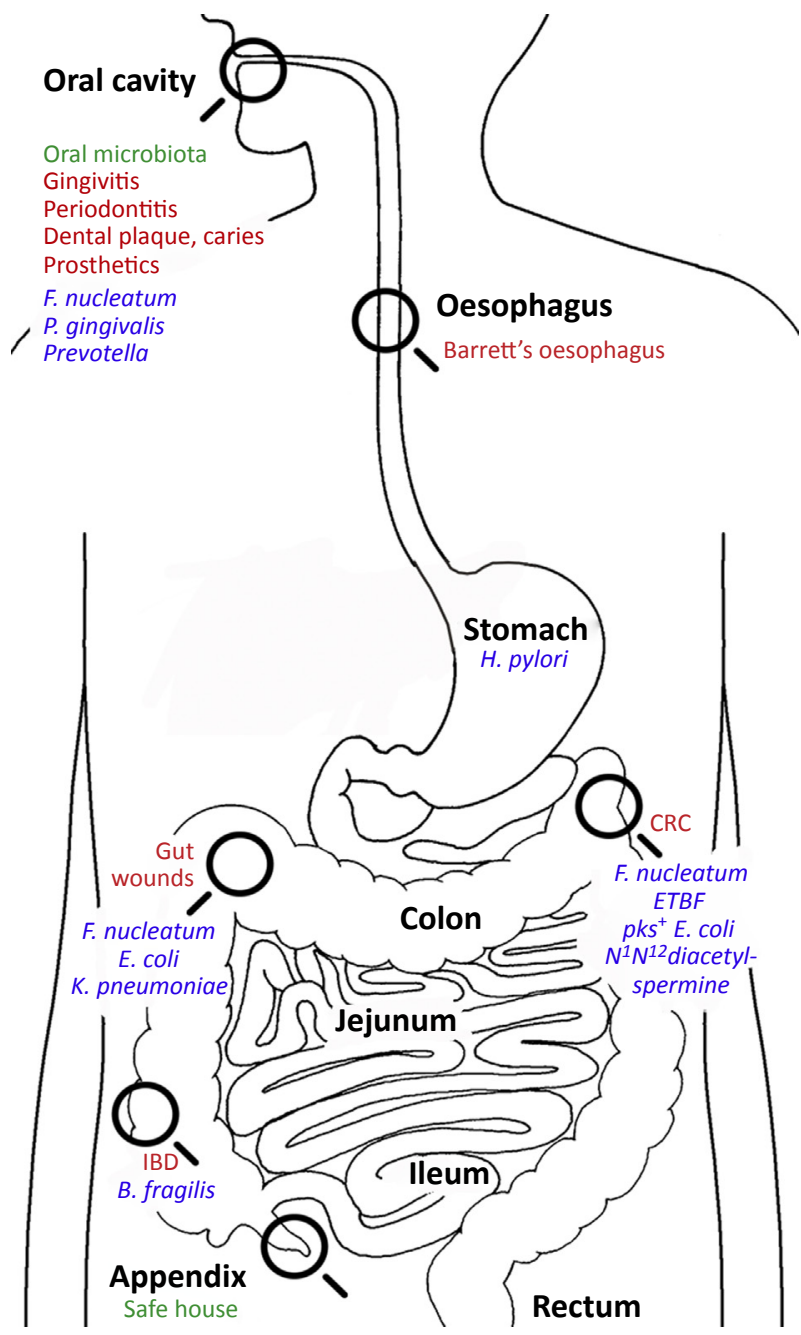

Trends in Microbiology

Figure 1. Biofilms can occur across the entire length of the orogastrointestinal tract. Healthy biofilms (indicated in green) are reported in the oral cavity and appendix, the latter serving as a bacterial safe house. Most reported biofilms in the orogastrointestinal tract are disease-linked (red). Driver species of pathogenic biofilm formation could be interesting biomarkers of the transition of a healthy to a diseased gut. Furthermore, bacterial surface and secreted molecules might serve as early-warning signals of the onset of disease (blue). Abbreviations: IBD, inflammatory bowel disease; CRC, colorectal cancer; ETBF, enterotoxigenic Bacteroides fragilis; E. coli, Escherichia coli; F. nucleatum, Fusobacterium nucleatum; H. pylori, Helicobacter pylori; K. pneumoniae, Klebsiella pneumoniae; P. gingivalis, Porphyromonas gingivalis.

\section{Glossary}

Adhesion: the event in which a bacterium attaches itself to its environment by interacting with receptors on the surface of the host using its surface molecules and appendages like pili (Box 1). Biofilms: matrix-enclosed mixed populations of bacteria and/or archaea that adhere to biotic and abiotic surfaces. Aggregates of bacteria embed themselves in a complex self-produced matrix of secreted polysaccharides. Once mature, bacterial cells can disperse to colonize new niches (Box 1). Biofilms are extremely resistant to environmental stresses and are an example of the collective behavior of bacteria (e.g., cross-feeding, gene transfer, pathogenicity, or antibiotic resistance).

Black Queen hypothesis: bacteria losing the ability to perform certain essential functions by relying heavily on other species in close proximity, in the sense that they even lose their own genetic capacity to perform these functions [5].

Colorectal cancer (CRC): cancer in the colon or rectum. CRC is the third most prevalent cancer worldwide and its incidence in young adults is increasing.

Gut wounds: damage to the intestinal mucosa that leads to a disruption of the intestinal epithelium, thus compromising its protective power to selectively interact with commensal and pathogenic bacteria. Gut wounds are often related to biofilm formation and the onset of more severe inflammatory diseases such as IBD $[45,47]$.

Immune exclusion: a specific immune response preventing an antigen from invading host tissue. Immune exclusion is involved in the prevention of bacterial translocation across the mucosal barrier, both by the presence of a lubricating mucus layer and the secretory immune system [18].

\section{Inflammatory bowel disease}

(IBD): inflammation of the gut in which the intestinal epithelium is compromised. Two main types are distinguished: Crohn's disease and ulcerative colitis.

Microcolony: small aggregates of adhering bacteria that protect themselves with a simple matrix from 


\section{Box 2. The Appendix as a Bacterial Safe House}

Current evidence supports the hypothesis that the appendix is more than just an evolutionary vestige. Its location in the intestinal tract, but shielded from peristalsis and transiently passing contaminants in the fecal stream, make the appendix an ideal safe house for commensal bacteria (Figure 1). If the colon is purged following pathogen exposure, infection, and antibiotic treatment, the appendix could aid in reseeding the colon and reinstating a healthy microbiota. The biofilm in this vermiform appendage is thought to protect its members from colonization with pathogens [16,56]. Recent research also pointed towards the close contact between the appendix and lymphatic tissue, rendering the appendix an important secondary immune organ promoting growth of some types of beneficial gut bacteria [56].

In industrialized countries with high hygiene standards, the appendix probably is less crucial. Given the lack of general outbreaks of enteric pathogens in these countries, the need for the reservoir function of the appendix is largely surpassed. Industrialized countries have a high rate of appendectomies, linked to a hyper reactivity of the immune system towards commensal bacteria [16], that is, the hygiene hypothesis. The exact effect of appendectomy on the constitution of the colon microbiota remains to be elucidated [57]. It would be informative to study the microbial population of the appendix in patients suffering from severe gut disorders to discern if the microbiota of the appendix is also affected. One might even speculate that repo(o)pulation of the appendix can become a form of therapy to ensure disease remission following drastic alterations of the intestinal microbiota. As appendices only occur in distinct species, and are, for instance, absent in mice, this forms an important obstacle in further research efforts in this direction.

pathogenic biofilms in the gut at the onset of disease [19]. Here, we address the evidence for biofilms both in healthy and diseased guts, and propose how mucosal biofilm development could be considered as a tipping point between health and disease. We also stress the importance for further studies addressing the manifestation of biofilms in both the healthy and diseased gastrointestinal tract.

\section{Biofilms in the Healthy State - An Ongoing Debate}

The ease of accessibility and noninvasive sampling have made oral biofilms a model for human biofilms. Both the healthy and diseased oral microbiome (dental caries, periodontitis, gingivitis, and oral cancer) are characterized by biofilms. Biofilm formation has also been observed on solid surfaces, such as in prosthetics and orthodontics $[14,15]$. These biofilms serve as a safe harbor for bacteria to reside in this highly versatile niche with varying temperature, $\mathrm{pH}$, redox, oxygen, salinity, nutrient concentrations, water flow, and oral hygiene [20]. Many in silico and in vitro models are available to describe the complex biofilm communities of the oral cavity in health and disease $[9,14]$. In the healthy oral microbiota, primary facultative anaerobic colonizers (mainly Gram-positives) are gradually replaced by Gram-negative anaerobic species, like Fusobacterium, Prevotella, and Porphyromonas [14] (Figure 1). The occurrence of both healthy and diseased stable oral microbiome communities offers the unique opportunity to assess disease onset and identify tipping points marking the transition between both.

In contrast to the generally accepted and corroborated presence of biofilms in the healthy oral microbiome, the situation in the gut is less straightforward and is topic of a lively debate. Some reports support the occurrence of mucosal biofilms in the healthy gut, which would benefit the host by promoting functions served by the microbiota, such as fortifying host defenses [7]. Mucosal biofilms can greatly increase bacterial residence time, hence stimulating bacteria-host synergy. Furthermore, it has been hypothesized that biofilms would enhance the exchange of nutrients between the microbiota and host [3]. Other indirect findings to support the presence of biofilms in a healthy gut include the slow growth rate of bacteria in the gut, increased plasmid transfer rates [17], expression of colonization factors, and the inference of colonization resistance by a healthy mucosal biofilm.

Although long thought to be dedicated to protect the host from pathogenic invasion, the gut immune system was found to also actively support the growth of specific commensal bacteria suboptimal environmental conditions (Box 1).

Organoid: miniature and simplified $3 \mathrm{D}$ version of an organ in vitro. Organoids are generated out of a few cells and offer a unique way to study biological processes as they enable us to investigate how cells interact within an organ and with the environment.

\section{Orogastrointestinal tract:}

combinatory term describing the oral cavity and the gastrointestinal tract, in concreto from mouth to rectum. Tipping point: intermediate unstable region between two alternative stable states of a system, where even the smallest fluctuations may lead to an abrupt shift to the alternative state [50,51]. 
[21]. This duality has been addressed in studies focusing on the role of secretory $\lg A(\operatorname{sg} A)$ and mucin. slgA is well known for its ability to 'cross-link' bacteria, that is, immune exclusion by agglutination, preventing translocation across the epithelial barrier, thus inhibiting formation of biofilms. Recent experimental data indicate that agglutination is achieved without any apparent specificity of slgA towards certain bacterial species [22], a finding supported by a recent study showing binding of slgA both to clear pathogens and to establish host-microbial symbiosis [23]. This supports the generic role of slgA in reducing the formation of intestinal biofilms. Apart from promoting agglutination, it has also been proposed that slgA stimulates the enchained growth of bacteria [24], thus restraining them from partaking in interactions with their environment. A biofilm-preventing role has also been proposed for mucin polymers that were found to prevent adhesion and aggregation of bacteria by retaining the cells in a planktonic state [25] and downregulating expression of biofilm-related genes in pathogens [26]. All of these studies indicate that $\lg A$ and mucin prevent the formation of biofilms. However, one study has proposed that slgA, together with mucin, can play a microbe-stimulating role by binding members of the 'normal, healthy' microbiota, thus supporting biofilm formation, that is, immune inclusion [18]. However, these and following studies of the same group were mainly performed in vitro with CaCo-2 cells, or ex vivo biopsies of appendix tissue using type 1 pili-producing pathogenic Escherichia coli as a model system that is barely representative of the gut microbiota [27-29]. With an improved methodology to preserve biofilms, the same group suggested that biofilms may occur in the proximal large human colon, supporting earlier microscopic observations [29,30]. However, these studies did not address healthy colonic tissue but rather focused on appendix tissue and showed biofilm formation in the appendices of humans, baboons, and rats [29,30]. Biofilm formation was reported to decrease progressively from the proximal to the distal end of the colon [16,30], that is, centering around the appendix. Based on the studies summarized above, experimental evidence for the presence of biofilms in healthy gut other than the appendix has not been provided. The appendix is a rudimental organ that is not in direct contact with the colonic luminal content. Recent findings support the hypothesis that the appendix serves as a safe house for human intestinal microbes, and here biofilm-like structures may have a function (Box 2, Figure 1).

Several reports mention the occurrence of small agglomerates of the gut microbiota, that is, microcolonies, in the gut [31-34]. Their formation is further supported by some major theoretical concerns that argue against the formation of thick biofilms in the gut [35]. These concerns include: the short transit time of intestinal content compared to the timescale of biofilm development [36], intrinsic properties of the mucus layer (e.g., a lubricating physical and selective barrier protecting intestinal epithelial cells) $[37,38]$, and the fact that known processes in the gut, including syntrophic interactions, can take place in the absence of biofilms [39]. The gut mucosa is a site of extremely high turnover and versatility, with recent data suggesting that the inner layer is sterile $[37,38,40]$. The absence of microbes in the mucus was earlier described and used as an argument against biofilm formation [41]. Consisting of heavily glycosylated proteins, mucus is a viscous gel-like substance reported to grow at a speed of $240 \mu \mathrm{m} / \mathrm{h}$ [42]. Epithelial cells are shed at a rate of 1-3 billion per hour in the small intestine and about 10 times slower in the colon. Another hampering factor is the constant propulsion of food and water by peristalsis [7].

In light of all current evidence and observations, we support a model in which the healthy mucosal microbiota establishes itself as microcolonies, and only in certain shielded areas of the gut, such as the appendix and potentially in some shielded crypts, mucosal biofilms could form (Box 2). These microenvironments of the gut render protection from the high flux of the lumen throughout the gut and enable an intimate relationship between the microbiome and the host. 
This model unifies all available experimental evidence and hypotheses both supporting and refuting the presence of mucosal biofilms. Further experimental evidence is, albeit challenging, crucial to substantiate the validity of this model (Box 3).

\section{Biofilms in Gut Disease}

Approximately $60-80 \%$ of infections in the human body are biofilm related [1]. Diseases of the orogastrointestinal tract are linked to a severe disturbance of the healthy microbiota. Biofilms containing potential pathogens seem to play an important role in the establishment of an alternative, disease-related microbiota by supporting host colonization via shielding from external stressors. Biofilms have been recognized to play a role in several conditions affecting the gut, including colorectal cancer (CRC), IBD, and gut wounds. More proximal to the gut, biofilms occur in stomach infections (Helicobacter pylori) [10], and in oral diseases like gingivitis and periodontitis [15].

Several studies, supported by microscopy data of clinical specimens, showed dense Bacteroides fragilis-dominated biofilms in patients with IBD, and sporadic manifestations of small bacterial communities (i.e., microcolonies) in healthy gut [11,43] (Figure 1). Notably, the mean density of the mucosal biofilm in IBD was found to be a 100-fold higher than in irritable bowel syndrome patients or healthy subjects [11]. IBD and other diseases severely affecting the gut are linked to a disruption of the healthy microbiota and mucosal epithelium. Loss of this crucial protective and selective layer facilitates species migration across the epithelial barrier and pathogenic biofilm outgrowth [38]. Biofilms offer a protective environment to pathogens and promote escape from host defense mechanisms, further facilitating disease manifestation [44].

Central to IBD is the formation of gut wounds, that is, severe damage to the intestinal mucosa due to inflammation leading to disruption of the intestinal epithelium. Aerobic and anaerobic microorganisms, including bacteria (e.g., Klebsiella pneumoniae, E. coli, Fusobacterium

\begin{abstract}
Box 3. Challenges in Microbiota Research
All arguments and speculations aside, solid experimental evidence on the occurrence and role of mucosal biofilms in the establishment of a healthy stable microbiota is scarce. This lack of experimental confirmation relates to some major practical challenges inherent to human microbiota research. A first challenge is the poor accessibility of the gastrointestinal tract. Sampling of the mucosal microbiota entails colonoscopy, endoscopy, or other invasive techniques, which are ethically not permitted in healthy subjects. Hence, there will be inevitably a bias towards analysis of compromised tissues in diseased patients. Some studies rely on the analysis of samples from apparently healthy parts flanking such compromised tissues $[11,12,43]$, but it remains to be evaluated how representative the biogeography in these tissues really is. This implies that most studies rely on fecal samples, introducing the second 'challenge' of microbiota research. Several studies have addressed the discrepancy in the constitution of the fecal microbiota, representing mainly the luminal and shed bacteria, versus the mucosa-associated microbiota [58]. Focus on the fecal microbiota also results in neglect of the spatial organization of intestinal bacterial communities.
\end{abstract}

Another way to study microbiota-host interaction is the use of animal models, with mice being the preferred one. Although widely used and insightful, the validity of mice models to address some conundrums of human microbiota research has been debated. The anatomy and architecture (e.g., absence of appendix, and enlarged caecum in mice), diet, metabolism, cell morphology, and environmental factors (housing, inbreeding etc.) are all significantly different when comparing humans to mice, together with a most notable dissimilarity in microbial and metagenome composition [59]. Mice and humans share many common genera in their microbiota, but these differ strongly in abundance. Indeed, only $4 \%$ of bacterial genes show considerable identity between the murine and human microbiota [60]. Extrapolation of results obtained in animal models to humans with respect to the microbiota composition and biogeography is thus not straightforward. A further alternative to bypass the need for biopsies from healthy persons and to cope with the physical inaccessibility of the gut, is the use of in vitro models of the human gut in health and disease [61]. As these in vitro gut systems are mostly seeded using fecal matter, results and conclusions of such studies need to be interpreted with caution. Organoids [54] and healthy biopsy tissue from preventive colonoscopies and endoscopies might offer opportunities to circumvent some of the common challenges of microbiota research, but need to be further established. 
nucleatum) and fungi, can colonize wounds in the gut and throughout the body $[45,46]$ (Figure 1). The surrounding microbiota in the oral cavity, skin, or gut form the primary source of potential infectious agents [45]. A breach in the epithelium impairs its ability to differentiate between beneficial, opportunistic, and pathogenic species, which leads to wound infection. If a biofilm of wound-colonizing bacteria is formed, wound healing is negatively affected. Tissue regeneration involves proliferation of intestinal epithelial cells and colonization by commensal bacteria (e.g., Akkermansia muciniphila and lactobacilli) to outcompete the wound-associated microbiota $[46,47]$.

Another severe biofilm-related condition affecting the gut is $\mathrm{CRC}$. A recent study showed that the establishment of $\mathrm{CRC}$ is strongly linked to biofilm formation. An invasive biofilm, harboring enterotoxigenic $B$. fragilis (ETBF) and $F$. nucleatum as key species, was detected in nearly all right-sided tumors and in 12\% of the left-sided tumors [48,49] (Figure 1). Interestingly, biofilms were also detected on tumor-free mucosal tissue distant from the actual tumor region [48].

Taken together, biofilms dominated by key pathogenic species play a key role in the establishment of gut diseases like gut wounds, $\mathrm{CRC}$, and IBD, and the shift towards a diseased microbiota.

\section{Biofilms as Tipping Points}

We hypothesize that the outgrowth of thick polymicrobial pathogenic mucosal biofilms marks the transition between two stable states: a healthy and diseased microbiota. Biofilms are the ideal environment for bacteria to establish virulence. The healthy ecological state of the microbiota, that is, commensal coexistence in microcolonies with the host, can be disrupted by environmental factors and pathogens supporting the outgrowth and transformation of healthy microbial consortia to pathogenic mature biofilms. These biofilms can withstand host defense systems and shift the microbiota to a deregulated state refractory to treatment. Our model fits well with the previously proposed tipping point theory [50].

The occurrence of mature biofilms on healthy tissue adjacent to $\mathrm{CRC}$ or IBD-affected tissue is an indication that biofilms may be an early-warning signal of the critical transition towards a disturbed, compromised, diseased gut. Biofilms containing potential pathogens on the gut mucosa are thus most probably tipping points [51]. Pivotal for further research is the identification of species that can be causally related to biofilm initiation and are indicative of a tipping point. Given their ubiquity in disease-related biofilms, F. nucleatum, ETBF, and by extension, $\mathrm{pks}^{+}$E. coli (implicated in familial adenomatous polyposis or FAP [19]) and other disease-driving pathogens can serve as early-warning signals of disease onset. Potential novel biomarkers include bacteria, quorum-sensing molecules, glycoproteins, and other bacterial surface molecules [52].

Detailed studies of an American and a Malaysian cohort and FAP patients substantiated the hypothesis that the occurrence of a pathogenic biofilm on the mucosa is a marker of CRC $[19,48,49]$. Investigation of the metabolome showed that polyamine metabolites in general, and $\mathrm{N}^{1}, \mathrm{~N}^{12}$-diacetylspermine in particular, were elevated both in the cancerous and surrounding normal tissue [53]. This study fits well with the here-postulated hypothesis of biofilms containing key pathogens being tipping points between two alternative stable states of the gut microbiota: healthy and diseased. The identification of these key pathogenic species and related metabolites can provide a wealth of novel biomarkers for the early diagnosis and targets for the treatment of various severe gut diseases. 


\section{Concluding Remarks}

How the microbiota establishes itself on the gut mucosa is of great interest. Many models have tried to solve this conundrum. Definite conclusions are hard to make due to technological challenges inherent in in situ microbiome research (Box 3). Apart from efforts to analyze its composition, however, mapping the structural components of the microbiota in time and space, that is, its biogeography, is crucial to fully grasp the functional dynamics of this complex community as well as the variable interaction with its environment. Sampling of healthy tissue could clarify the occurrence of mucosal biofilms in the healthy gut. Whilst organoids hold promise to improve in vitro studies [54], the use of biopsy tissues from colonoscopy and endoscopy examinations of healthy subjects can push the field forward. The development of alternative sampling techniques that do not harm the patient will be key to study the spatial organization of the microbiota in situ.

Although adhesion events, microcolony and biofilm formation are difficult to distinguish, the mucosal microbiota is most likely to manifest itself as microcolonies, whereas the likelihood of mature biofilms in the healthy mucosa is low. Biofilms may occur in shielded areas of the gut, such as the appendix, which functions as a bacterial safe house (Box 2).

Mature, thick polymicrobial biofilms containing pathogens have been established as important features of disease, for example, chronic gut wounds, IBD, and CRC. Studies of the latter indicate that mucosal pathogenic biofilms might be used as a biomarker for the onset of disease. In view of pathogenic biofilm outgrowth as a tipping point between healthy and diseased states, one can assume that outgrowth of mucosal biofilms in seemingly healthy patients may be an early-warning signal of disease. Although further research is required to substantiate this model, biofilms including pathogenic species can be hypothesized to be tipping points between two alternative states: healthy and diseased gut (see Outstanding Questions). Other promising biomarkers of disease-related biofilm formation are key biofilm pathogens (e.g., F. nucleatum) and their associated virulence factors and metabolites (e.g., polyamines).

Further research is necessary to show the validity of the here-proposed model and to once and for all end the discussion on the biogeography of the microbiota in the gut. The current evidence suggests that key species and molecules can be identified and linked to distinct disease states of the gut microbiota, thus offering potential for diagnostic and therapeutic purposes.

\section{Acknowledgments}

The authors want to acknowledge Dr Annelies Geirnaert and Dr Miguelangel Cuenca for critical comments. This research was supported by an ERC grant Microbes Inside (250172), a Spinoza Award and the Soehngen and the Soehngen Institute of Anaerobic Microbiology (SIAM Gravity Grant 024.002.002), funded by The Netherlands Organization for Scientific Research (NWO) awarded to WMdV. HT holds a Marie Sklodowska-Curie fellowship of the European Union's Horizon 2020 research and innovation programme under agreement No 703577 (Glycoli) to support her work at ETH Zurich. FN is supported by the NWO Veni project 016.Veni.181.092.

\section{References}

1. Costerton, J.W. et al. (1995) Microbial biofilms. Annu. Rev. Microbiol. 49, 711-745

2. Stoodley, P. et al. (2002) Biofilms as complex differentiated communities. Annu. Rev. Microbiol. 56, 187-209

3. Hooper, L.V. and Gordon, J.I. (2001) Commensal host-bacterial relationships in the gut. Science 292, 1115-1118

4. Otto, M. (2014) Physical stress and bacterial colonization. FEMS Microbiol. Rev. 38, 1250-1270

5. Morris, J.J. et al. (2012) The Black Queen Hypothesis: evolution of dependencies through adaptive gene loss. mBio 3, e00036-00012

6. Balcazar, J.L. et al. (2015) The role of biofilms as environmental reservoirs of antibiotic resistance. Front. Microbiol. 6, 1216
7. Sonnenburg, J.L. et al. (2004) Getting a grip on things: how do communities of bacterial symbionts become established in our intestine? Nat. Immunol. 5, 569-573 metabolism in the large intestine. J. Appl. Microbiol. 102, 1197-1208

9. Filoche, S. et al. (2010) Oral biofilms: emerging concepts in microbial ecology. J. Dent. Res. 89, 8-18

10. Garcia, A. et al. (2014) Biofilm and Helicobacter pylori: from environment to human host. World J. Gastroenterol. 20, 5632-5638

11. Swidsinski, A. et al. (2005) Spatial organization and composition of the mucosal flora in patients with inflammatory bowel disease. J. Clin. Microbiol. 43, 3380-3389
8. Louis, P. et al. (2007) Understanding the effects of diet on bacterial

\section{Outstanding Questions}

How to define clear cut-offs distinguishing adhesion events from microcolony and biofilm formation? This is necessary to enable scalability and uniform reporting on such events in complex niches.

How does the healthy intestinal microbiota manifest itself? Where and when does the microbiota occur as an agglomerate of microcolonies or as a thin, low-complexity biofilm?

How can we deal with the inaccessibility of the healthy human mucosa to provide answers to questions pertaining to the biogeography of the healthy microbiota?

Is the establishment of thick pathogenic polymicrobial mucosal biofilms marking the onset of disease a common theme across niches and pathologies? Can longitudinal studies confirm the role of such biofilms as tipping points between a healthy and diseased mucosa?

How do important gut-regulatory molecules, such as slgA, mucus, and its proteins, play a role in the establishment of the microbiota in health and disease?

Can species driving the shift of a healthy to a diseased microbiota, and their associated metabolites and surface molecules, be exploited as biomarkers and early-warning signals of disease onset? And what avenues does this open towards novel therapeutics?

How can this field be developed from correlation to causality? 
12. Swidsinski, A. et al. (2005) Bacterial biofilm within diseased pancreatic and biliary tracts. Gut 54, 388-395

13. Macfarlane, S. et al. (2007) Microbial colonization of the upper gastrointestinal tract in patients with Barrett's esophagus. Clin. Infect. Dis. 45, 29-38

14. Wake, N. et al. (2016) Temporal dynamics of bacterial microbiota in the human oral cavity determined using an in situ model of dental biofilms. NPJ Biofilms Microbiomes 2, 16018

15. Zarco, M.F. et al. (2012) The oral microbiome in health and disease and the potential impact on personalized dental medicine. Oral Dis. 18, 109-120

16. Bollinger, R.R. et al. (2007) Biofilms in the large bowel suggest an apparent function of the human vermiform appendix. J. Theor. Biol. 249, 826-831

17. Licht, T.R. et al. (1999) Plasmid transfer in the animal intestine and other dynamic bacterial populations: the role of community structure and environment. Microbiology 145, 2615-2622

18. Everett, M.L. et al. (2004) Immune exclusion and immune inclusion: A new model of host-bacterial interactions in the gut. Clin. Appl. Immunol. Rev. 4, 321-332

19. Dejea, C.M. et al. (2018) Patients with familial adenomatous polyposis harbor colonic biofilms containing tumorigenic bacteria. Science 359, 592-597

20. Avila, M. et al. (2009) The oral microbiota: living with a permanent guest. DNA Cell Biol. 28, 405-411

21. Dishaw, L.J. et al. (2016) Gut immunity in a protochordate involves a secreted immunoglobulin-type mediator binding host chitin and bacteria. Nat. Commun. 7, 10617

22. Planer, J.D. etal. (2016) Development of the gut microbiota and mucosal IgA responses in twins and gnotobiotic mice. Nature 534, 263-266

23. Donaldson, G.P. et al. (2018) Gut microbiota utilize immunoglobulin A for mucosal colonization. Science 360, 795-800

24. Moor, K. et al. (2017) High-avidity IgA protects the intestine by enchaining growing bacteria. Nature Published online April 12, 2017. http://dx.doi.org/10.1038/nature22058

25. Caldara, M. et al. (2012) Mucin biopolymers prevent bacterial aggregation by retaining cells in the free-swimming state. Curr. Biol. 22, 2325-2330

26. Kavanaugh, N.L. et al. (2014) Mucins suppress virulence traits of Candida albicans. mBio 5, e01911

27. Bollinger, R.R. et al. (2003) Human secretory immunoglobulin A may contribute to biofilm formation in the gut. Immunology 109 , 580-587

28. Bollinger, R.R. et al. (2006) Secretory IgA and mucin-mediated biofilm formation by environmental strains of Escherichia coli: role of type 1 pili. Mol. Immunol. 43, 378-387

29. Palestrant, D. et al. (2004) Microbial biofilms in the gut: visualization by electron microscopy and by acridine orange staining. Ultrastruct. Pathol. 28, 23-27

30. Bollinger, R.R. et al. (2007) Biofilms in the normal human large bowel: fact rather than fiction. Gut 56, 1481-1482

31. Banwell, J.G. et al. (1985) Intestinal microbial flora after feeding phytohemagglutinin lectins (Phaseolus vulgaris) to rats. Appl. Environ. Microbiol. 50, 68-80

32. Macfarlane, S. et al. (2011) Mucosal biofilm communities in the human intestinal tract. Adv. Appl. Microbiol. 75, 111-143

33. Macfarlane, S. et al. (1997) Consequences of biofilm and sessile growth in the large intestine. Adv. Dent. Res. 11, 59-68

34. Donelli, G. et al. (2012) Biofilm-growing intestinal anaerobic bacteria. FEMS Immunol. Med. Microbiol. 65, 318-325

35. de Vos, W.M. (2015) Microbial biofilms and the human intestinal microbiome. NPJ Biofilms Microbiomes 1, 15005

36. Conway, T. and Cohen, P.S. (2015) Commensal and pathogenic Escherichia coli metabolism in the gut. Microbiol. Spectr. Published online June 2015. http://dx.doi.org/10.1128/microbiolspec.MBP-0006-2014

37. Johansson, M.E. et al. (2011) The two mucus layers of colon are organized by the MUC2 mucin, whereas the outer layer is a legislator of host-microbial interactions. Proc. Natl. Acad. Sci. U. S. A. 108 (Suppl. 1), 4659-4665

38. Johansson, M.E. et al. (2014) Bacteria penetrate the normally impenetrable inner colon mucus layer in both murine colitis models and patients with ulcerative colitis. Gut 63, 281-291

39. Stams, A.J. and Plugge, C.M. (2009) Electron transfer in syntrophic communities of anaerobic bacteria and archaea. Nat. Rev. Microbiol. 7, 568-577

40. Johansson, M.E. et al. (2015) Normalization of host intestinal mucus layers requires long-term microbial colonization. Cell Host Microbe 18, 582-592

41. Swidsinski, A. et al. (2007) Comparative study of the intestinal mucus barrier in normal and inflamed colon. Gut 56, 343-350

42. Gustafsson, J.K. et al. (2012) An ex vivo method for studying mucus formation, properties, and thickness in human colonic biopsies and mouse small and large intestinal explants. Am. J. Physiol. Gastrointest. Liver Physiol. 302, G430-G438

43. Swidsinski, A. et al. (2002) Mucosal flora in inflammatory bowel disease. Gastroenterology 122, 44-54

44. Hoarau, G. et al. (2016) Bacteriome and mycobiome interactions underscore microbial dysbiosis in familial Crohn's disease $\mathrm{mBi}$ Published online September 20, 2016. http://dx.doi.org/ 10.1128/mBio.01250-16

45. Bertesteanu, S. et al. (2014) Polymicrobial wound infections: pathophysiology and current therapeutic approaches. Int. J. Pharm. 463, 119-126

46. Scales, B.S. and Huffnagle, G.B. (2013) The microbiome in wound repair and tissue fibrosis. J. Pathol. 229, 323-331

47. Alam, A. et al. (2016) The microenvironment of injured murine gut elicits a local pro-restitutive microbiota. Nat. Microbiol. 1, 1502

48. Dejea, C.M. et al. (2014) Microbiota organization is a distinct feature of proximal colorectal cancers. Proc. Natl. Acad. Sci. U. S. A. 111, 18321-18326

49. Drewes, J.L. et al. (2017) High-resolution bacterial 16S rRNA gene profile meta-analysis and biofilm status reveal common colorectal cancer consortia. NPJ Biofilms Microbiomes 3, 34

50. Scheffer, M. et al. (2009) Early-warning signals for critical transitions. Nature 461, 53-59

51. Lahti, L. et al. (2014) Tipping elements in the human intestinal ecosystem. Nat. Commun. 5, 4344

52. Tytgat, H.L. and de Vos, W.M. (2016) Sugar coating the envelope: glycoconjugates for microbe-host crosstalk. Trends Microbiol. 24, 853-861

53. Johnson, C.H. et al. (2015) Metabolism links bacterial biofilms and colon carcinogenesis. Cell Metab. 21, 891-897

54. Zachos, N.C. et al. (2016) Human enteroids/colonoids and intestinal organoids functionally recapitulate normal intestinal physiology and pathophysiology. J. Biol. Chem. 291, 3759-3766

55. Burmolle, M. et al. (2014) Interactions in multispecies biofilms: do they actually matter? Trends Microbiol. 22, 84-91

56. Smith, H.F. et al. (2017) Morphological evolution of the mammalian cecum and cecal appendix. C. R. Palevol 16, 39-57

57. Girard-Madoux, M.J.H. et al. (2018) The immunological functions of the Appendix: An example of redundancy? Semin. Immunol. $36,31-44$

58. Eckburg. P.B. et al. (2005) Diversity of the human intestinal microbial flora. Science 308, 1635-1638

59. Nguyen, T.L. et al. (2015) How informative is the mouse for human gut microbiota research? Dis. Model Mech. 8, 1-16

60. Hugenholtz, F. and de Vos, W.M. (2018) Mouse models for human intestinal microbiota research: a critical evaluation. Cell. Mol. Life Sci. 75, 149-160

61. McDonald, J.A. et al. (2015) Simulating distal gut mucosal and luminal communities using packed-column biofilm reactors and an in vitro chemostat model. J. Microbiol. Methods $108,36-44$ 